\title{
Effects of ZNF139 on gastric cancer cells and mice with gastric tumors
}

\author{
HONG-FENG NIE ${ }^{1}$, YONG LI ${ }^{2}$, ZHEN-XING LI ${ }^{3}$, JI-XING MU ${ }^{1}$ and JIN-SHENG WANG ${ }^{1}$ \\ ${ }^{1}$ Department of General Surgery, The First Affiliated Hospital, Xingtai Medical College, Xingtai, Hebei 054001; \\ ${ }^{2}$ Department of General Surgery, The Fourth Affiliated Hospital, Hebei Medical University, Shijiazhuang, Hebei 050011; \\ ${ }^{3}$ Department of General Surgery, Hebei United University Affiliated Hospital, Tangshan, Hebei 063000, P.R. China
}

Received April 3, 2015; Accepted May 26, 2016

DOI: $10.3892 / \mathrm{ol} .2016 .4982$

\begin{abstract}
Gastric cancer (GC) is the fourth most common type of cancer, worldwide. The major molecular factors associated with the pathogenesis of GC remain unclear. Previous studies found that zinc finger proteins are highly abundant in human eukaryotes and tissues, and play an important role in maintaining normal cellular functions and have an association with tumor initiation. In the current study, interference technology was used to silence the ZNF139 protein, a zinc finger protein that was previously found to be closely associated with GC. The results showed that cell viability and proliferation were inhibited in the Znf139-knockdown of GC cells. Additional study found that the expression levels of B cell lymphoma-2 (Bcl-2) and survivin messenger RNAs and proteins were downregulated in Znf139-silenced cells, indicating that cells expression Znf139 are able to induce the growth of tumor cells by mediating the apoptosis pathway. Further in vivo experiments showed that Znf139 knockdown downregulated the expression levels of Bcl-2 and survivin in mice. Overall, the in vitro and in vivo findings of the present study indicate that ZNF139 may be actively involved in the progression of GC.
\end{abstract}

\section{Introduction}

Gastric cancer (GC) is the fourth most common type of cancer and ranks as the second most common cause of cancer-associated mortalities, while the incidence rate is gradually rising every year worldwide (1). Although certain advances, such as combination chemotherapy, have been achieved in chemotherapy in recent years, the therapeutic effect remains unsatisfactory (2). Numerous molecular factors associated with pathogenesis of GC have been reported, but the key factors remain unclear. Therefore, finding out the controlling factors of GC and their effects

Correspondence to: Dr Yong Li, Department of General Surgery, The Fourth Affiliated Hospital, Hebei Medical University, 12 Jiankang Road, Shijiazhuang, Hebei 050011, P.R. China

E-mail: li_yong_hbyd@163.com

Key words: zinc finger protein 139, gastric cancer, survivin, B cell lymphoma-2, apoptosis on its pathogenesis may increase the current understanding of the mechanisms underlying gastric carcinogenesis and facilitate the development gene-based therapies.

Zinc finger proteins are highly abundant in human eukaryotic cells and tissues and are important for the maintenance of normal cellular functions (3). As previously reported, numerous members of the zinc finger protein family are associated with the initiation, development, invasion and metastasis of tumors, including ZNF165 in urinary bladder cancer and ZNF217 in colorectal adenoma and ovarian cancer (4,5). ZNF139, a member of the kruppel family of zinc-finger-containing transcription factors, was found to be closely associated with GC in a previous study (6). However, the mechanism of ZNF139 in the growth and metastasis of GC cells was not clarified. Therefore, the aim of the present study is to detect the specific impact and the mechanism of ZNF139 on GC tumor cells by using interference technology, orthotopic transplantation and immunohistochemistry.

\section{Materials and methods}

Cell culture. The human GC SGC7901 cell line was purchased from The Cell Bank of Type Culture Collection of Chinese Academy of Sciences (Shanghai, China) and were cultivated in RPMI-1640 medium (Gibco; Thermo Fisher Scientific, Inc., Waltham, MA, USA) containing $10 \%$ fetal bovine serum (Gibco; Thermo Fisher Scientific, Inc.), penicillin (100 unit/ml) and streptomycin $(100 \mu \mathrm{g} / \mathrm{ml})$. The tumor cells were cultured in a humidified atmosphere of $5 \% \mathrm{CO}_{2}$ at $37^{\circ} \mathrm{C}$.

Plasmid construction and transfection. The vector RNAi-Ready pSIREN-RetroQ-ZsGreen plasmid was obtained from Clontech Laboratories, Inc. (Mountainview, CA, USA). The expression vectors containing either the anti-Znf139 small interfering RNA (siRNA) sequence ACCTCGGAAGATTCAGCAT for the negative control (scramble) sequence GACGAGTTGACT GCGATTG were constructed, as previously described (7), and termed Znf139-siRNA or siRNA-scramble expression vector, respectively. Znf139-siRNA expression vector (Znf139-siRNA group), siRNA-scramble expression vector (siRNA-scramble group) or blank expression vector (mock group) were transfected into SGC7901 cells using Lipofectamine 2000 (Invitrogen; Thermo Fisher Scientific, Inc.), according to the manufacturer's 
instructions. A fluorescence microscope was used to observe the transfection efficiency at $24 \mathrm{~h}$ after transfection and $80 \%$ transfection efficiency was considered to be successful transfection. Transfection efficiency was calculated as follows: Transfection efficiency $(\%)=$ (number of fluorescent cells/total number of cells) x 100 .

\section{3-(4,5-dimethyl-thiazol-2-yl)-2,5-diphenyl-2H-tetrazolium} bromide (MTT) assay. SGC7901 cells $\left(1 \times 10^{4}\right.$ per well) were planted into 96-well microtiter plates and incubated overnight prior to being transfected with siRNA. At $24 \mathrm{~h}$ subsequent to transfection, $20 \mu \mathrm{l}$ MTT (Sigma-Aldrich, St. Louis, MO, USA) assay solution $(5 \mathrm{mg} / \mathrm{ml})$ was added into the wells for $4 \mathrm{~h}$ at $37^{\circ} \mathrm{C}$. After discarding the medium, the formazan crystals were dissolved in dimethyl sulfoxide (DMSO) and the absorbance was measured at $490 \mathrm{~nm}$ on a microplate reader (Bio-Rad Laboratories, Inc., Hercules, CA, USA).

5-ethynyl-2'-deoxyuridine (EdU) assay. Briefly, cells $\left(1 \times 10^{4}\right.$ cells per well) were seeded into 96 -well plates with $100 \mu \mathrm{l} \mathrm{EdU} \mathrm{(50} \mu \mathrm{M}$; Sigma-Aldrich) added, and cultured in an incubator for $2 \mathrm{~h}$ at room temperature. The cells were fixed with $4 \%$ paraformaldehyde for $30 \mathrm{~min}$, treated with glycine $(2 \mathrm{mg} / \mathrm{ml})$ for $5 \mathrm{~min}$ and washed with phosphate-buffered saline (PBS) three times. Subsequently, $100 \mu \mathrm{l} 1 \mathrm{X}$ Apollo ${ }^{\circledR}$ reaction cocktail (Sigma-Aldrich) was added to the cells to react for $30 \mathrm{~min}$ in the dark, and 1X Hoechst 33342 (Sigma-Aldrich) was used to stain cell nuclei for $30 \mathrm{~min}$ at room temperature. The nuclear morphology was then observed with an immunofluorescence microscope and the percentage of EdU-labeled (red) cells was noted.

Reverse transcription-quantitative polymerase chain reaction (RT-qPCR). Total RNA was extracted from SGC7901 cells using Trizol reagent (Invitrogen; Thermo Fisher Scientific, Inc.) following the manufacturer's protocol. RNA $(2 \mu \mathrm{g})$ was then reverse transcribed into complementary DNA (cDNA) using the SuperScript Reverse transcriptase kit (Invitrogen; Thermo Fisher Scientific, Inc.). RT-qPCR was performed with the SYBR Premix Ex Taq II (Takara Bio, Inc., Otsu, Japan) on an ABI-7500 Sequence Detection System (Applied Biosystems; Thermo Fisher Scientific, Inc.). Housekeeping gene $\beta$-actin was chosen as an internal control to detect the relative expression of the target genes. The thermal cycling conditions were $95^{\circ} \mathrm{C}$ for $30 \mathrm{sec}$ and $58^{\circ} \mathrm{C}$ for $1 \mathrm{~min}$. The sequences of the PCR primers were as follows: Znf139 forward, CTTCCTGAGTTCTTGGTT TCG and reverse, CCTTTGACCCACTGGTTTATG; $B c l-2$ forward, GGTGGGGTCATGTGTGTGG and reverse, CGG TTCAGGTACTCAGTCATCC; survivin forward, GGACCG CCTAAGAGGGCGTGC and reverse, AATGTAGAGATG CGGTGGTCCTT; $\beta$-actin forward, CATGTACGTTGCTAT CCAGGC and reverse, CTCCTTAATGTCACGCACGAT. The comparative quantification $(\mathrm{Cq})$ cycle method $\left(2^{-\Delta \Delta \mathrm{Cq}}\right)$ was used to calculate relative expression levels of Znf139/ $\beta$-actin, Bcl-2/ $\beta$-actin and survivin/ $\beta$-actin (8).

Western blot analysis. The cells were lysed using a ReadyPrep Protein Extraction kit (Bio-Rad Laboratories, Inc.) for 5 min. After removing cell debris by centrifugation at $12,000 \mathrm{x} \mathrm{g}$ for
$15 \mathrm{~min}$, the supernatant containing the proteins was collected and boiled in sample buffer for $5 \mathrm{~min}$. The concentrated proteins were separated by $10 \%$ sodium dodecyl sulfate polyacrylamide gel electrophoresis so that the specific proteins could be transferred onto polyvinylidene fluoride membranes (Roche Diagnostics, Basel, Switzerland). The membranes were blocked using PBS containing 0.05\% Tween 20 and 5\% skim milk for $1 \mathrm{~h}$ at room temperature. After being washed 3 times in Tris-buffered saline containing Tween 20, the membranes were incubated with rabbit anti-mouse ZNF139 polyclonal (1:800 dilution; cat no. ab126124; Abcam, Cambridge, UK), Bcl-2 monoclonal (1:800 dilution; cat no. ab32124; Abcam), survivin polyclonal (1:800 dilution; cat no. ab469; Abcam) or $\beta$-actin polyclonal (1:5,000 dilution; cat no. ab8227; Abcam) primary antibodies overnight at $4^{\circ} \mathrm{C}$, and then exposed to goat anti-rabbit horseradish peroxidase-conjugated secondary antibody (1:5,000 dilution; cat no. 074-1506; KPL, Inc., Gaithersburg, MD, USA). Immunoreactive bands were detected by the enhanced chemiluminescence system (GE Healthcare Life Sciences, Little Chalfont, UK).

Mouse model establishment. Establishment of the GC tissue orthotopic transplantation mouse model was separated into 2 steps. Firstly, subcutaneous tumor-transplanted mouse model was established using 6-8 weeks old male BALB/C (nu/nu) nude mice. All mice were acclimated to a natural day-night cycle at $21^{\circ} \mathrm{C}$, and given free access to food and water for 1 week prior to the trial. These nude mice were divided into 2 groups: Group A was injected with Znf139-knockdown SGC7901 cells and group B was injected with SGC7901 cells transfected with siRNA-scramble. After the mice were subcutaneously injected with $7 \times 10^{4}$ cells per mouse on the backside, the mice were kept in specific pathogen free conditions for 3 weeks until the implanted tumor grew to $1 \mathrm{~cm}$ diameter. The mice were anesthetized with $6 \%$ pentobarbital sodium and sacrificed by cervical dislocation. Then the tumor tissues were removed, sliced into 1x1x1 mm pieces and implanted to the backside of other nude mice. The source of the orthotopic transplantation was coming from the sixth generation subcutaneously-transplanted tumor slices. For the second step, the establishment of the orthotopic transplanted mouse model, nude mice were anesthetized with $6 \%$ pentobarbital sodium. A 5-10 $\mathrm{mm}$ abdominal incision was made along the left paramedian line in order to expose the gastric wall. The serosa of the greater curvature of stomach was cut so that the tumor slice could be implanted. OB glue (1-2 drops; Guangzhou Baiyun Medical Adhesive Co., Ltd., Baiyun, China) was used to seal the rupture and the enterocoelia was closed using a suture. Mice were weighed daily. After 90 days, all mice were sacrificed by cervical dislocation due to the development of systemic failure, and the tumors excised from stomach were measured using a vernier caliper and analytical balance. The procedures for the care and use of animals were approved by the Ethics Committee of the Fourth Hospital of Hebei Medical University (Shijiazhuang, Hebei) and all applicable institutional and governmental regulations concerning the ethical use of animals were followed.

Statistical analysis. Statistical analysis was performed using SPSS software (version 17.0; SPSS Inc., Chicago, IL, USA). Data were analyzed by one-way analysis of variance and are 


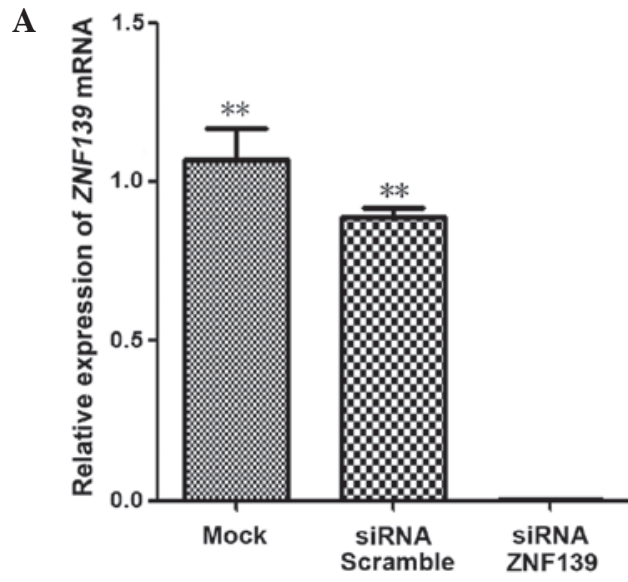

B

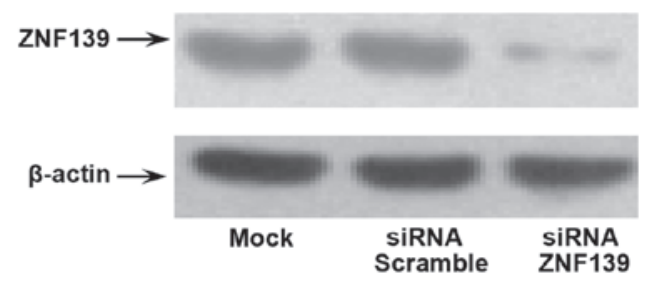

Figure 1. Expression of ZNF139 mRNA and protein levels in the gastric cancer SGC7901 cell line subjected to Znf139-siRNA. Cells were transfected with $100 \mathrm{nM}$ Znf139-siRNA for $48 \mathrm{~h}$ and the expression of ZNF139 was identified by (A) reverse transcription-quantitative polymerase chain and (B) western blot analysis. The expression of ZNF139 was significantly increased in Znf139-siRNA cells versus cells in the mock and siRNA-scramble group. ${ }^{* *} \mathrm{P}<0.01$ vs. siRNA-ZNF139. ZNF139, zinc finger protein 139; mRNA, messenger RNA; siRNA, small interefering RNA.

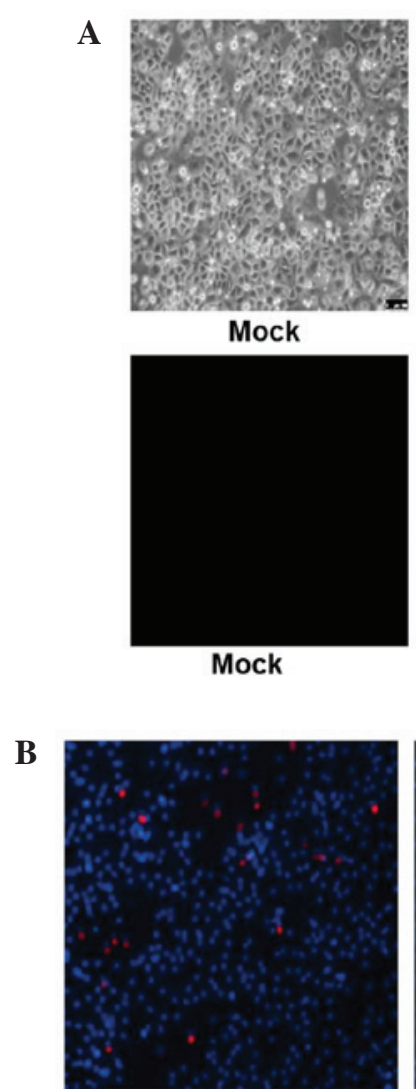

Mock

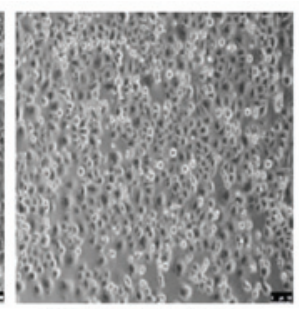

SiRNA-Scramble

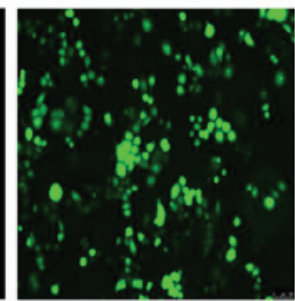

SiRNA-Scramble

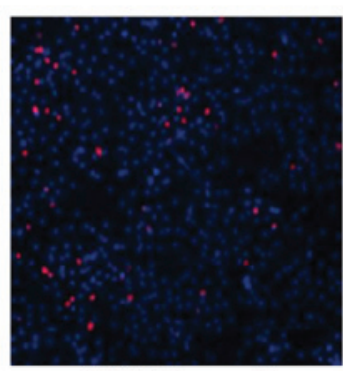

SIRNA-Scramble

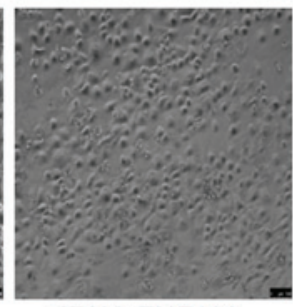

SiRNA-ZNF139

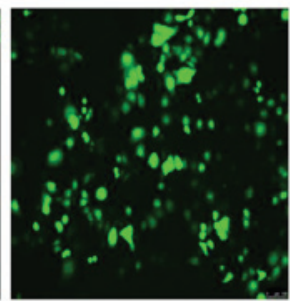

SIRNA-ZNF139

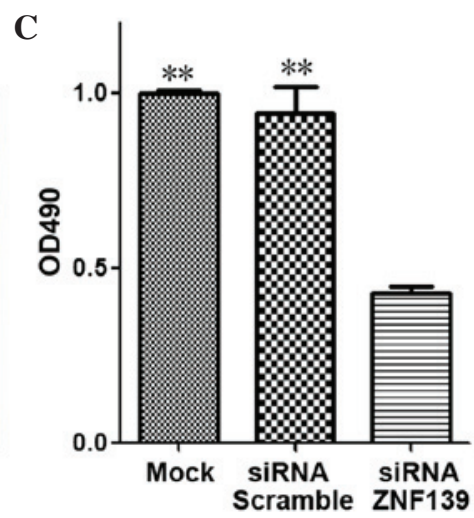

Figure 2. Effect of Znf139 on SGC7901 cell growth. SGC7901 cells were transfected with 100 nM Znf139-siRNA for 48 h. (A) Growth activity of SGC7901 cells was significantly inhibited in the cells transfected with Znf139-siRNA compared with the negative and the blank groups. (B) 5-ethynyl-2'-deoxyuridine and (C) 3-(4,5-dimethyl-thiazol-2-yl)-2,5-diphenyl-2H-tetrazolium bromide assays were performed to test the effect of Znf139 on cell growth. Cell growth was significantly decreased in Znf139-siRNA cells versus cells in the mock and siRNA-scramble group ${ }^{* *} \mathrm{P}<0.01$ vs. siRNA-ZNF139. siRNA, small interefering RNA; ZNF139, zinc finger protein 139; OD, optical density.

expressed as the mean \pm standard deviation. All experiments were repeated three times. Multiple comparison between the groups was performed using the Student-Newman-Keuls method. $\mathrm{P}<0.05$ was considered to indicate a statistically significant difference.

\section{Results}

Effect of siRNA on Znf139 expression in SGC7901 cells. To demonstrate the role of ZNF139 on tumor growth and apoptosis, a ZNF139 RNA interference vector was 

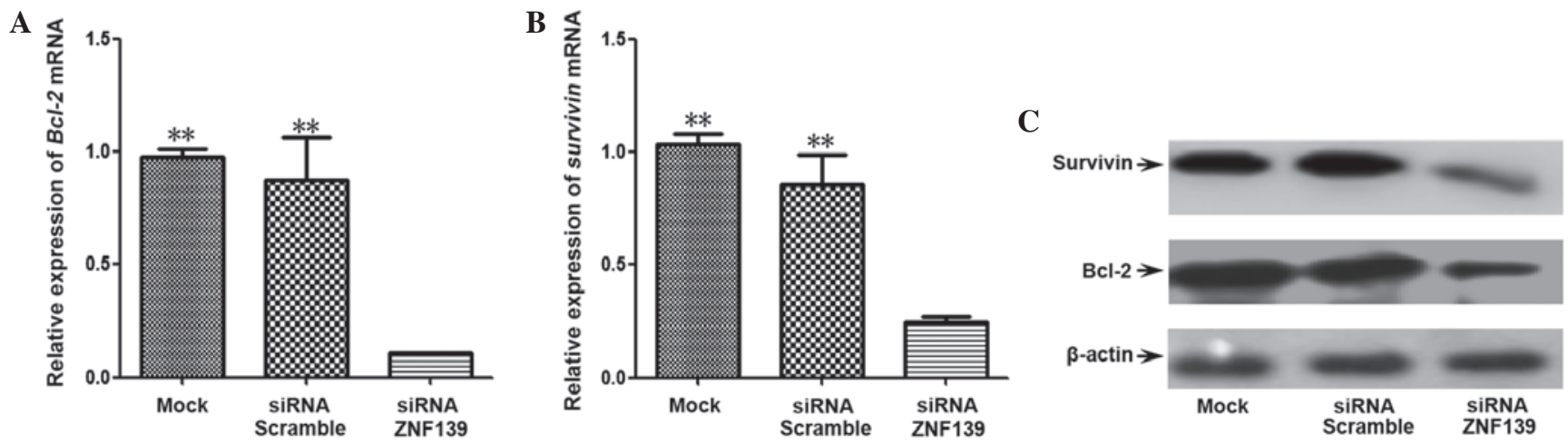

Figure 3. Effect of Znf139 on the expression of Bcl-2 and survivin in SGC7901 cells. Cells were transfected with Znf139-siRNA or control scramble-siRNA. The mRNA expression levels of (A) Bcl-2 and (B) survivin were detected by reverse transcription-quantitative polymerase chain reaction and the (C) Bcl-2 and survivin protein levels were detected by western blot analysis. The expression levels of Bcl-2 and survivin were significantly decreased in Znfl39-siRNA cells versus cells in the mock and siRNA-scramble group. ${ }^{* *} \mathrm{P}<0.01$ vs. siRNA-ZNF139. ZNF139, zinc finger protein 139; siRNA, small interfering RNA; mRNA, messenger RNA; Bcl-2, B-cell lymphoma-2.

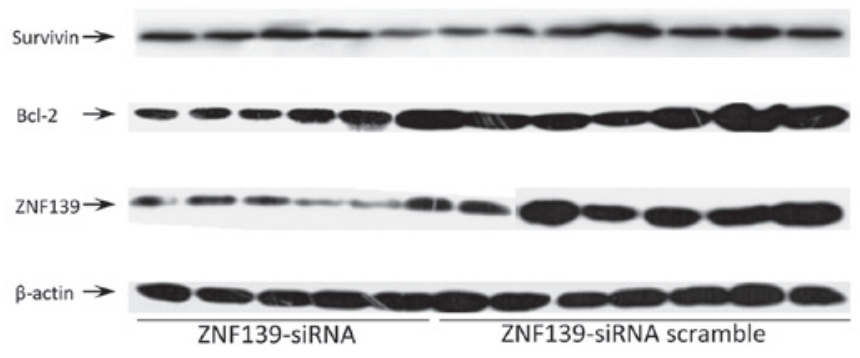

Figure 4. Effects of silencing ZNF139 on the expression of Bcl-2 and survivin in mouse models. The expression of ZNF139 was markedly inhibited in Znf139-siRNA cells compared with cells in siRNA-scramble group, and the expression levels of Bcl-2 and survivin were significantly decreased in Znf139-siRNA cells compared with cells in the siRNA-scramble group. ZNF139, zinc finger protein 139; Bcl-2, B-cell lymphoma-2.

constructed with green fluorescent protein transfected into the GC SGC7901 cell line. In the cells transfected with Znf139-siRNA, the expression of ZNF139 was evidently decreased in the messenger RNA (mRNA) (Fig. 1A) and protein (Fig. 1B) compared with the siRNA-scramble and mock control groups (all $\mathrm{P}=0.001$ ). There was no significant difference between the siRNA-scramble and mock control groups (Fig. 1). These results indicated that the constructed Znf139-siRNA expression vector was able to inhibit the transcription and translation of endogenous Znfl39 efficiently.

Effect of Znf139-siRNA on cell growth. Further experiments were performed to detect the effect of ZNF139 on cell growth. SGC7901 cells were transfected with Znf139-siRNA and siRNA-scramble. As shown in Fig. 2, the growth of Znf139-knockdown cells was inhibited significantly after $48 \mathrm{~h}$ transfection (Fig. 2A). Subsequently, EdU and MTT assays were used to confirm whether cell viability and proliferation were inhibited following the knockdown of Znf139. According to the EdU analysis, the proliferation of the Znf139-knockdown cells was evidently inhibited compared with the siRNA-scramble and mock control groups (Fig. 2B). As shown in the MTT assay, the viability of Znf139-silenced cells was evidently inferior compared with the siRNA-scramble and mock control groups $(\mathrm{P}=0.001$ and
$\mathrm{P}=0.003$, respectively; Fig. $2 \mathrm{C}$ ). These results suggest the Znfl39 gene is associated with the growth and proliferation of gastric tumor cells.

Effect of Znf139-siRNA on cell apoptosis. To further investigate the association between ZNF139 and cell apoptosis, the $B c l-2$ and survivin genes were detected with qPCR and western blot analysis. The results showed that the expression levels of $\mathrm{Bcl}-2$ and survivin mRNAs $(\mathrm{P}=0.001$ and $\mathrm{P}=0.002$, respectively) and proteins were downregulated in Znf139-silenced cells compared with the siRNA-scramble and mock groups (Fig. 3). These data indicated that Znf139 was able to induce the growth of tumor cells by mediating the apoptosis pathway.

Znfl39 knockdown downregulated the expression of Bcl-2 and survivin in mice. Through weighing the mice daily, after the establishment of the human GC nude mouse orthotopic transplantation model, the mice appeared to experience systemic failure, showing symptoms of emaciation, roachback, listlessness and hypoactivity by 90 days. The mice were then sacrificed by cervical dislocation and the tumors excised from stomach were measured using a vernier caliper and analytical balance. The tumors in mice orthotopically transplanted with Znf139-knockdown SGC7901 cells were significantly inhibited compared with those orthotopically transplanted with siRNA-scramble SGC7901 cells, and there was no metastasis of the tumor in peritoneum and lymph node in mice orthotopically transplanted with Znf139-knockdown SGC7901 cells. The western blot analysis results revealed that the expression levels of Bcl-2 and survivin were significantly decreased in the tumors of orthotopic transplant mice that interfered with Znfl39 siRNA (Fig. 4), which was in agreement with the results of the in vitro experiment.

\section{Discussion}

GC is one of the most common digestive system malignant tumors worldwide, which is associated with a high fatality rate, particularly in Asia. Therefore, researching the mechanism 
of GC is of great importance (9). Various molecular factors have been be shown to be associated with the pathogenesis of GC, but the major genes affecting the growth and prognosis of GC tumors remains unclear. The GC SGC7901 cell lines were moderately differentiated adenocarcinoma cells, which are commonly found in clinical patients (10). ZNF139, a member of the zinc finger protein family, is a regulating factor at transcription level (6). Dekken et al first discovered the increased expression of ZNF139 in an adenocarcinoma of the esophago-gastric junction (11). A previous study found that ZNF139 could affect gastric tumors by promoting the expression of fascin and heterogeneous nuclear ribonucleoproteins A2/B1 (12). In addition, the expression of ZNF139 was found to be closely associated with $\mathrm{GC}$, indicating that ZNF139 may be involved in gastric carcinogenesis and development (12). Another previous study showed that ZNF139 was involved in GC multidrug resistance by simultaneously promoting the expression of multidrug resistance protein 1 (MDR1), multidrug resistance-associated protein 1 (MRP1) and $\mathrm{Bcl}-2$, and inhibiting Bcl-2-associated X protein (13). In order to explore the association between ZNF139 and the growth of tumor cells, the expression of ZNF139 was silenced in SGC7901 cells by first applying RNA interference technology. As shown in the results, the viability and proliferation were extremely suppressed in the SGC7901 cells with Znf139 knockdown, indicating that the inhibition of ZNF139 was conducive to the growth of GC cells in vitro.

To investigate the influence of ZNF139 in vivo, a human GC nude mouse orthotopic transplantation model was created, which could simulate GC biological behavior, including proliferation, invasion and metastasis, in the human body. The orthotopic transplantation model of GC is stable, reliable and considered to be the most optimal model of GC so far. In the orthotopic transplantation mice with Znfl39 knockdown, the growth of the tumor was evidently suppressed and there were signs of no lymphatic metastasis, suggesting that ZNF139 could affect the development and metastasis of GC tumors in vivo. In order to explore which genes are regulated by Znf139, changes of apoptosis-associated genes were detected in the cells and mice of each group. The Bcl-2 and survivin genes, the representative genes of the apoptotic pathway, were significantly downregulated following Znf139 knockdown, including their protein products. Previous studies found that Bcl-2 enhances the anti-apoptotic ability of the tumor cells by regulating the mitochondrial redox state of thiol (14). Survivin inhibits caspase activation, and therefore leads to the negative regulation of programmed cell death (15). In summary, these results show that the expression of anti-apoptotic associated genes, $B c l-2$ and survivin, was extremely reduced following the silencing of Znf139. This indicates that ZNF139 could facilitate the growth and metastasis of $\mathrm{GC}$ by promoting $\mathrm{Bcl}-2$ and survivin.

In the present study, ZNF139 was demonstrated to affect the growth and metastasis of GC tumors by inhibiting cell apoptosis. The establishment of a human GC nude mouse orthotopic transplantation model partly enabled the simulation of GC biological behavior the in human body. However, the anatomical structure and microcirculatory system are varied in the human body and nude mice, in addition to differences in the immunodeficiency of nude mice. Recent studies have shown that ZNF139 may be associated with multiple drug resistance by regulating the MDR1 and MRP1 genes (13). Therefore, future studies are recommended to focus on building animal models that are closer to the internal environment of the human body, as well as exploring the association between ZNF139, MDR1 and gene therapy in GC.

\section{Acknowledgements}

The present study was supported by The National Natural Science Foundation of China, Beijing, China (grant no. 81072033).

\section{References}

1. Ma Z, Wang W, Jin G, Chu P and Li H: Effect of statins on gastric cancer incidence: A meta-Analysis of case control studies. J Cancer Res Ther 10: 859-865, 2014.

2. Imamura T, Komatsu S, Ichikawa D, Kubota T, Okamoto $\mathrm{K}$, Konishi H, Shiozaki A, Fujiwara H, Morimura R, Murayama Y, et al: Poor prognostic subgroup in T3N0 stage IIA gastric cancer, suggesting an indication for adjuvant chemotherapy. J Surg Oncol 111:221-225, 2015.

3. Wei S, Zhang L, Zhou X, Du M, Jiang Z, Hausman GJ, Bergen WG, Zan L and Dodson MV: Emerging roles of zinc finger proteins in regulating adipogenesis. Cell Mol Life Sci 70: 4569-4584, 2013.

4. Singh PK, Srivastava AK, Dalela D, Rath SK, Goel MM and Bhatt ML: Frequent expression of zinc-finger protein ZNF165 in human urinary bladder transitional cell carcinoma. Immunobiology 220: 68-73, 2015.

5. Li P, Maines-Bandiera S, Kuo WL, Guan Y, Sun Y, Hills M, Huang G, Collins CC, Leung PC, Gray JW and Auersperg N: Multiple roles of the candidate oncogene ZNF217 in ovarian epithelial neoplastic progression. Int J Cancer 120: 1863-1873, 2007.

6. Li Y, Tan BB, Zhao Q, Fan LQ, Wang D and Liu Y: ZNF139 promotes tumor metastasis by increasing migration and invasion in human gastric cancer cells. Neoplasma 61: 291-298, 2014.

7. Zhang S, Yang JH, Guo CK and Cai PC: Gene silencing of TKTL1 by RNAi inhibits cell proliferation in human hepatoma cells. Cancer Lett 253: 108-114, 2007.

8. Livak and Schmittgen: Analysis of relative gene expression data using real-time quantitative PCR and the $2-\Delta \Delta \mathrm{Ct}$ method. Methods 25: 402-408, 2001.

9. Fock KM: Review article: The epidemiology and prevention of gastric cancer. Aliment Pharmacol Ther 40: 250-260, 2014.

10. Zhang S, Zuo L, Gui S, Zhou Q, Wei W and Wang Y: Induction of cell differentiation and promotion of endocan gene expression in stomach cancer by melatonin. Mol Biol Rep 39: 2843-2892, 2012.

11. van Dekken H, Tilanus HW, Hop WC, Dinjens WN, Wink JC, Vissers KJ and van Marion R: Array comparative genomic hybridization, expression array, and protein analysis of critical regions on chromosome arms 1q, 7q, and 8p in adenocarcinomas of the gastroesophageal junction. Cancer Genet Cytogenet 189: 37-42, 2009.

12. Li Y, Tan BB, Fan LQ, Zhao Q, Song ZC and Wang D: Proteomic identification and comparison of differentiation-related proteins in gastric carcinoma cell lines. Zhonghua Zhong Liu Za Zhi 32: 179-184, 2010 (In Chinese).

13. Li Y, Tan BB, Zhao Q, Fan LQ, Liu Y and Wang D: Regulatory mechanism of ZNF139 in multi-drug resistance of gastric cancer cells. Mol Biol Rep 41: 3603-3610, 2014.

14. Roy MJ, Vom A, Czabotar PE and Lessene G: Cell death and the mitochondria: Therapeutic targeting of the BCL-2 family-driven pathway. Br J Pharmacol 171: 1973-1987, 2014.

15. Rubio N, Garcia-Segura LM and Arevalo MA: Survivin prevents apoptosis by binding to caspase-3 in astrocytes infected with the BeAn strain of Theiler's murine encephalomyelitis virus. J Neurovirol 18: 354-363, 2012. 